\title{
Supporting collaboration with technology: does shared cognition lead to co-regulation in medicine?
}

\author{
Susanne P. Lajoie • Jingyan Lu
}

Received: 14 January 2009 / Accepted: 9 May 2011 /

Published online: 25 May 2011

(C) Springer Science+Business Media, LLC 2011

\begin{abstract}
The theoretical distinctions between metacognition, self-regulation and selfregulated learning are often blurred which makes the definition of co-regulation in group learning situations even more difficult. We have started to explore co-regulation in the context of decision making in simulated emergencies where medical teams work together to manage patient cases. Our earlier work has described the relationship between collaborative decision-making in this context as well as discourse patterns that emerge in a simulated medical emergency (Lu \& Lajoie, 2008). This paper examines the interactions that occur during this simulation that reflect the relationship between coregulation and medical decision-making. There are two collaborative learning conditions, a traditional situation where the instructor facilitates collaboration by using a whiteboard to document the group's construction of a medical argument (the traditional whiteboard condition, TW). The second condition uses technology to facilitate the collaboration, where individuals use laptops and an interactive whiteboard (IW) where they can interact with the problem list as it is being created. Our assumption was that the IW would facilitate communication beyond the teacher-student, to include student-student both within and between the various subgroups. The IW group could document their medical arguments by using a structured template for constructing, annotating and sharing arguments. We found that participants in the IW condition differed from the TW condition in that they engaged in more adaptive decision-making behavior early on in the intervention. Similar overall levels of metacognitive activity were found in both conditions but the pattern and timing of metacognitive categories varied. Specifically, the IW group engaged in more planning and orienting than the TW group at the outset of the problem. Early engagement and co-regulation occurred in the IW group which led to shared understandings and subsequently to effective patient management in latter sessions $(11.5 \%$ vs. $3.6 \%$ in TW). Technology supported greater metacognitive activity
\end{abstract}

S. P. Lajoie $(\triangle)$

McGill University, 3700 McTavish Street, Montreal, Canada, QC H3A 1Y2

e-mail: susanne.lajoie@mcgill.ca

J. Lu

The University of Hong Kong, Pokfulam, Hong Kong 
overall (44\% vs $29 \%$ in the non supported group). Furthermore, technology facilitated greater planning $(23 \%$ vs. $10 \%)$ and orienting $(10 \%$ vs $1 \%)$ early in the medical problem solving activity. We refer to specific indicators in the discourse that help operationalize the concept of co-regulation.

Keywords Collaboration · Medical decision making $\cdot$ Distributed cognition $\cdot$ Situated learning $\cdot$ Simulations

The usefulness of self-regulation in learning contexts is well documented. However, the literature blurs the distinctions between constructs of metacognition, self-regulation, and self-regulated learning (Dinsmore et al. 2008; Lajoie 2008). Given this ambiguity, defining co-regulation among multiple learners becomes a challenge. This paper takes on this challenge in the context of describing co-regulation in simulated medical emergencies. In particular, we look at differences between teams that are supported by technology and those who are not supported and examine the level of metacognitive activity in both teams and how such activity leads to decision making about how to help a patient in distress. We provide the theoretical underpinnings of metacognition and co-regulation followed by the medical context where we study these processes in action.

\section{Metacognition, self-regulation and co-regulation}

Flavell (1971) defines metacognition as thinking about one's own thinking. He states that through experience we learn what to monitor, how to set goals to achieve understanding, use strategies to achieve our goals and ultimately evaluate our successes and failures. As we develop we learn what knowledge to monitor, and discover mechanisms for how to selfregulate (Baker and Brown 1984). In addition, both cognitive and affective dimensions of regulation become apparent in the context of the environment where it was studied (Bandura 1982, 1986). As the body of literature on metacognition grew, so did the refinement in how it was studied. In particular, self-regulation became of interest in the context of specific learning situations and integrated theories of self-regulated learning (SRL) arose whereby cognitive, motivational, behavioral and contextual factors were taken into consideration (Corno and Mandinach 1983; Pintrich 2004; Zimmerman 1989).

Students are self-regulated to the degree that they are cognitively, motivationally, and behaviorally active participants in their own learning processes (Zimmerman 1989, 2001) and efficiently manage their own learning (Boekaerts et al. 2000; Butler and Winne 1995; Paris and Paris 2001; Schunk and Zimmerman 1994; Winne 2001; Winne and Perry 2000; Zimmerman and Schunk 2001). Recent research shows that when students lack selfregulatory skills their ability to learn from open-ended learning environments is undermined (Azevedo et al. 2004).

The relationship between metacognition, self-regulation, and co-regulation is complex. Although metacognition and self-regulation imply self-directed learning, such learning may still need to be guided by other viewpoints. A key mechanism in improving metacognition or self-regulation is the ability to observe and listen to other perspectives. Even though metacognition is considered an individual phenomenon there is a social aspect to it (Salonen et al. 2005). Hacker and Bol (2004) refer to the fact that private cognitions are influenced by social experiences and vice versa. Social constructivist learning theories speak to these interrelationships whereby co-regulation refers to the process by which our 
social environment serves to support or scaffold individual participation and learning (McCaslin 2004). Groups of individuals can be seen as multiple self-regulating agents that socially regulate each other's learning (Volet et al. 2009).

Salonen et al. (2005) propose that co-regulation requires awareness of one's own metacognitive experiences about the task at hand as well as that of partners engaging in the task. In order to understand multiple perspectives in social contexts communication is needed (Bartsch and Wellman 1995) as well as social cognition (Lories et al. 1998) where socio-cognitive and social-cultural (Zimmerman 2004) influences are most relevant to metacognition (Hacker and Bol 2004). Salonen et al. (2005) describe the complex interconnections between the participants' sociocognitive and affective behaviors. Considerations must be made with respect to both the content-related communications and the affective responses that are most often discovered in the context of negotiating relational or command positions (who is more dominant). Collaboration and co-regulation may decline when there are imbalances that occur in the group due to insufficient understanding of the semantic content of the exchanges or if there is a mismatch in the relational balance, i.e., one person being too dominant or submissive in the learning exchange.

Co-regulation and collaboration have sometimes been discussed interchangeably when evidence of convergence, synthesis, or shared understanding occurs in problem solving (Reusser 2001). However, Volet et al. (2009) argue for refinements in the definition of coregulation. These refinements would be operationalized along a continuum of social regulation from "individual regulation within group" to "co-regulation as a group". Volet et al. provide criteria for looking at the relationship between social regulation and content processing. They state that high-level content-processing can be observed within an individual or group as engagement in elaborating, interpreting, reasoning, building on ideas, explaining in one's own words, or help seeking for understanding. Low-level contentprocessing might be seeking help for details or facts, reading verbatim from text. Individual regulation features one speaker, other than simple acknowledgements from others (e.g., "yep", "uh huh"), whereas co-regulation represents verbal contributions from multiple group members. Volet et al. provide guidance for coding regulation on both a content and social dimension that could be a promising direction for different content contexts.

The role of affect is another element that should be considered in situations where coregulation is required. Olekalns and Smith (2005) describe how positive affect can lead to trustful partnerships that lead to joint gains whereas the opposite occurs in situations where negative characterizations occur. Their analysis consisted of retrospective think-alouds using videos of dyad interactions to stimulate recall of metacognitive activity. Their analysis revealed that specific turning points or events precipitated change in the nature of the mock job offer negotiation between dyads who were asked to role-play an employer or employee where both parties were instructed to maximize value for themselves. Turning points were described as substantive (offer, interest), attitudinal (positive characterization, negative characterization), and procedural (positive procedural, negative procedural). The results demonstrated that both cognitive and affective trust are increased when a negotiator identifies interest, positive characterization, and positive procedural turning points in the other party's behaviors. The negotiator's ability to build joint gain was influenced by the number and type of turning points, with interest turning points leading to the greatest joint gain and negative characterization turning points leading to the lowest joint gain.

In collaborative learning situations a coordinated continuous effort must be made to achieve a shared understanding of the situation at hand (Roschelle and Teasley 1995). When imbalances occur in either meaning-making or in the social relationship then coordinated self and co-regulation is needed to re-establish common ground. Co-regulation 
involves a fine balance between cognition, communication and emotion in the context of the task at hand.

Communication is often the source of data when studying co-regulation. However, when technology supports the collaboration then technology is also an artifact of the situation in the same way that the actual task is part of the overall context in which co-regulation is studied. In the section below, the task, emergency medicine, and the technology that supports medical decision making, are described.

\section{Medical decision making in simulated emergencies}

The Joint Commission on Accreditation of Healthcare Organizations (2006) reported that the most frequent cause of medical errors leading to death or serious adverse outcomes could be attributed to communication errors. Successful teamwork requires both effective communication and coordination (Wright et al. 2009). Naturalistic decision-making situations, where individuals must share their cognition to be effective, are good contexts to study co-regulation given that communication and coordinated efforts are needed. Wright et al. (2009) examined teamwork in classroom based patient assessment tasks and in a simulated emergent care situation and found a stronger relationship between strong teamwork and clinical performance in the simulated setting. Wright et al.'s definitions stem from teamwork research on military aircrews (Bowers et al. 1993) and health care teams (Baker et al. 2005) where teamwork is described in terms of levels of assertiveness, decisionmaking, situation assessment, leadership, and communication. Although, we do not analyze teams in terms of leadership roles we do see parallels for exploring decision-making based on the notion of situation awareness which points to what Salonen et al. (2005) referred to as awareness of the task at hand, of one's own understanding and that of others.

Medical decision making often involves collaboration and team members must communicate and share their awareness of a situation (situation-specific metacognitive skills) in order to establish shared mental models of the task (Orasanu 2005). Shared mental models have been described as shared understandings of task goals and task relevant knowledge (Cannon-Bowers et al. 1993). Examples of shared mental models comes from analyses of how air crews work effectively and efficiently in times of high stress (Klein et al. 1993). Team members develop the shared understandings needed to make the decisions required to achieve long-term goals through explicit communication. Shared models guide daily activities and reduce the need for continual explicit directions. Shared mental models help define problems, acceptable outcomes, and roles of team members. Mental models may evolve with time and in particular groups must establish shared situation awareness that relies on common understandings of dynamically changing situations (Cannon-Bowers et al. 1993). In emergent medical situations, doctors must assess and communicate rapidly changing patient conditions to other medical personnel for various reasons. This in turn facilitates the construction of shared understandings of goals, plans, and actions for managing the patient effectively. Building up dynamic shared mental models and shared situation awareness requires both metacognition and co-regulation.

\section{The deteriorating patient simulation}

This study explores the role that technology played in supporting metacognition and coregulation in the context of a simulated medical emergency activity termed the 
"deteriorating patient" (Wiseman and Snell 2008). The deteriorating patient activity simulates medical emergencies that students will encounter in internal medicine. Participants are placed in situations where they must make decisions quickly based on the patient's rapidly deteriorating medical condition. The teacher plays two roles, one as the deteriorating patient and the other as the duty nurse where he responds to student actions as they play the role of the physician. As the deteriorating patient, the teacher acts out the patient's fluctuating physical conditions. As the duty nurse he constantly updates patient's fluctuating vital signs and symptoms. The students' job is to stabilize the patient by stopping the deterioration of the vital signs and returning them to normal values by discovering the immediate cause of the trouble. If they do the right thing the patient will recover; if they do nothing or they do the wrong thing the patient will become sicker. As the role-play progresses the patient will decline more quickly if students fail to solve the problem. Students ask the nurse questions about the patient and based on his answers determine what to do next. The nurse carries out their orders, reports results and updates any ensuing changes in the patients' vital signs and symptoms. When the students need help they ask other students to step in to solve the problem. The instructor scaffolds the students by what he says in his role as the duty nurse or as the patient. He provides hints within the context of their decision-making by providing feedback as a nurse or as a patient. Students learn to ask the right questions, order the correct tests, and manage the situation.

The teacher usually conducted the deteriorating patient activity with small classes where each student played the role of the physician, while other students observed, or stepped in when an impasse was reached. We wanted to reduce the bystander effect and increase the level of student engagement in this activity by introducing collaboration, where instead of one student acting as the physician, a team of 2 or 3 students would collaborate in the physician role, while other students would listen and discuss alternatives in their own teams. Collaborative learning provides students opportunities for developing social and communication skills and promote deeper level of thinking and shared understanding (Johnson and Johnson 1999). In analyzing the discourse we could examine whether such shared understanding was achieved and what types of metacognitive statements each team made and what the relationship was to patient management outcomes.

We were also interested in how technology could support the collaboration throughout the role-play activity. Situated cognition takes place within a nexus of activities, tools, and culture (Brown et al. 1989). The introduction of technology-supported collaboration tools were designed to allow teams to share their thoughts about the patient case by creating and sharing annotations to the white board, thereby increasing the types of external representations that would support the problem solving activity. Visualizing the team process by external means was predicted to provide a more effective means for coregulation. In this particular study we investigate whether or not technological support of communication and argumentation during team problem solving leads to more metacognition and co-regulation and better decision-making in the form of patient management. More specifically, do technology-supported teams demonstrate higher levels of metacognitive and co-regulation activity than teams without technology support?

In a previous study (Lu \& Lajoie, 2008) we investigated communicative activities and medical decision making in the context of the deteriorating patient activity. In one condition students used a traditional whiteboard to document medical arguments on how to solve a medical emergency. In the other condition students used interactive whiteboards where they could document their medical arguments by using a structured template for constructing, annotating and sharing arguments. The interactive whiteboard was meant to scaffold 
collaborative decision-making processes by promoting productive discussions of tentative actions and plans as well as providing a structured patient chart where students could comment on the decisions of other participants in the activity and participate by proposing alternative moves. Students who had the benefit of the interactive whiteboard engaged in more adaptive decision-making behavior early on in the intervention. This early engagement led to shared understandings and productive argumentation. In doing so, the interactive whiteboard facilitated effective patient management.

Although this previous study captured the nature of decision-making it did not examine instances of metacognition or co-regulation in the context of communicative activities. Because cognitive and metacognitive strategies are closely intertwined and dependent upon each other, any attempt to examine one without acknowledging the other would not provide an adequate picture. Decision-making processes (e.g., planning, collecting data, managing, and interpreting) parallel the metacognitive control processes (e.g., planning, executing, orientation, and elaboration). In particular, planning and orienting have similarities, as do managing and executing. However, even if the cognitive processes overlap, the purpose of metacognitive strategies are to monitor the effectiveness of decision-making processes and alter such processes when they fail. There has been growing interest in the research literature in regards to investigating the deployment of metacognitive processes within the context of collaborative problem solving based on the assumption that the context could foster the use of metacognitive strategies that are critical in solving problems (see Iiskala et al. 2011). The co-regulated aspects of the problem-solving task are addressed in this paper by using speaker turn as our unit of analysis, to determine whether learners collaborating with each other would facilitate an individual learners attempt to monitor and evaluate the problem-solving process. The broader implications of better capturing the onset of co-regulation is to inform teachers in terms of what, when, and how to scaffold learners during problem-solving. Our premise is that studying co-regulation is critical to determining what, when, and how to scaffold medical decision making in the context of the deteriorating patient activity. The identification of whether or not students are monitoring and evaluating their medical decision-making in the context of the deteriorating patient activity determines whether or not they engage in remedial strategies (i.e., based on learners' metacognitive knowledge and standards). In the event that learners decision making (e.g., planning, collecting data, managing, and interpreting) is altered on the basis of their collaborative efforts to monitor and evaluate the problem solving process, then learners enacted a metacognitive control process that reflects the knowledge and practices of that discipline (e.g., planning, executing, orientation, and elaboration).

In analyzing the discourse we examine whether such shared understanding was achieved and what types of metacognitive statements each team made and what the relationship was to patient management outcomes. Visualizing the team process by external means was predicted to provide a more effective means for co-regulation. Specifically, an individual's problem-solving process was articulated in an organized manner through the external representation provided by the interactive whiteboard, thereby enabling others to support his or her efforts to monitor and evaluate the problem-solving process. In this particular study we investigate whether or not technological support of communication and argumentation during team problem solving leads to more metacognition and coregulation and better decision-making in the form of patient management. More specifically, do technology-supported teams demonstrate higher levels of metacognitive and co-regulation activity in the form of monitoring and evaluation than teams without technology support? 


\section{Methodology, design and analysis}

\section{Subjects and design}

Fourteen third year medical students were recruited from an internal medicine rotation. The students attended two clinical teaching sessions: one at the beginning and one at end of their 2-month rotation. The teacher organized students into two conditions (one we refer to as the traditional whiteboard condition, TW, that had no technology support, and one we refer to as the interactive whiteboard condition, IW, with technological support). Each condition had three teams that worked together as units during the teaching sessions where they were asked to participate in the deteriorating patient activity. Each condition had a traditional whiteboard at the front of the class that the teacher used to document the patient's problem list as the medical decisions were being communicated by the medical teams. However, the IW condition used EBEAM software to interface information between the traditional whiteboard and their individual laptops that were used in their teams (see Fig. 1). The IW provides a form of groupware that enables groups of participants to collaborate for a common purpose (Khoshafian and Buckiewicz 1995) by making collaborations and communications visible. The difference between the two conditions was that in the IW condition the students could annotate the medical argument on their laptops, adding and sharing information they thought relevant to solving the medical problem. The TW teams communicated about the patient's current situation and plans for patient management among each other before they played the active role as the doctor. However, in the TW condition the teacher added the information to the board as each team verbalized next steps.

The teacher presents the patient case and asks for a volunteer team to start solving the problem. When the team runs into difficulty, the teacher tells them to call the junior resident (played by another team). When the junior resident reaches an impasse s/he calls the senior

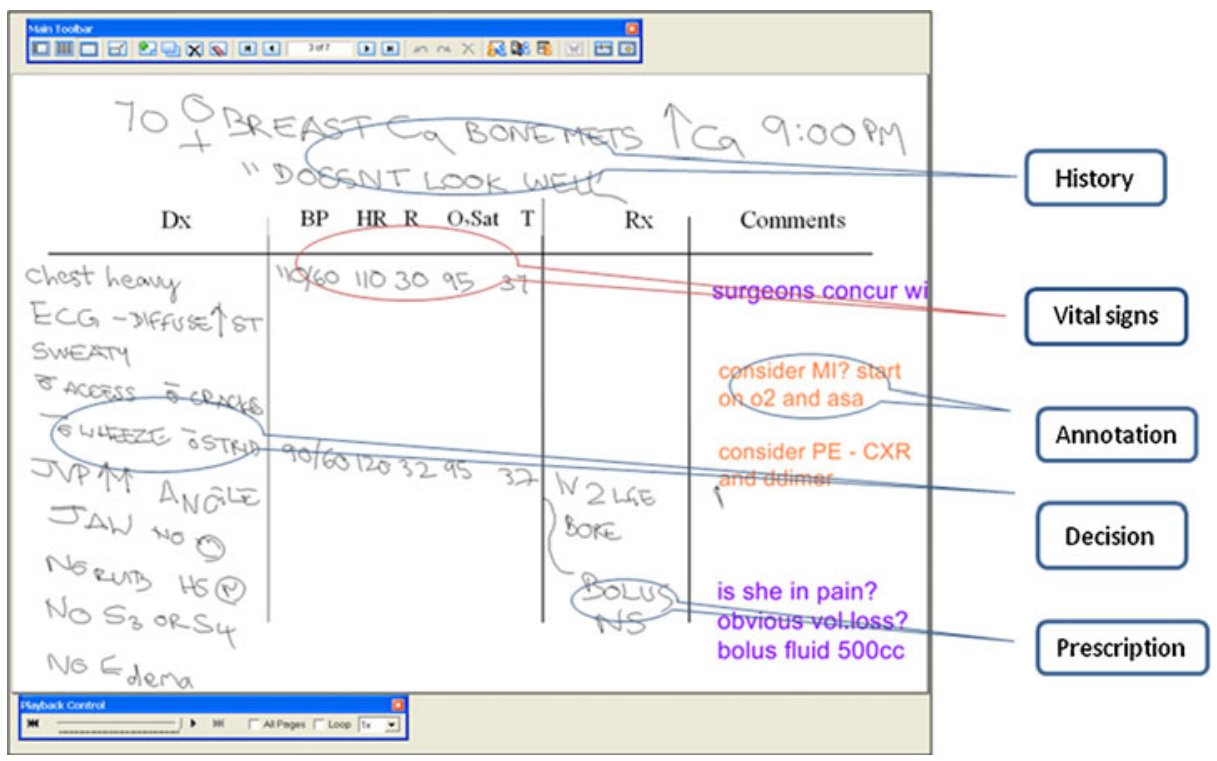

Fig. 1 Screenshot of interactive whiteboard 
resident who is played by a third team. In this way the activity simulates the operation of a real medical emergency, where participants ask for consults.

Audio and video records of discourse during the deteriorating patient activity were collected to characterize and compare student metacognition and co-regulation in the two conditions. A qualitative analysis was conducted to describe patterns in the two conditions. The entire protocol of deteriorating patient activity for each condition was examined over time in the problem solving sequence, where time was determined by segmenting the protocols into thirds that represented early, middle and late problem solving activity. Both conditions were equivalent in that the same procedures and activities were used with teams. We were comparing conditions rather than relationships between teams and thus dependency between teams was a non-issue. Time was an equivalent measure for both conditions. We were interested in the presence, amount, and timing of metacognitive discourse in both conditions. We also examine metacognitive activity with respect to coregulation and effective decision-making regarding patient management in the TW and IW conditions.

We adapted the coding scheme described by Meijer et al. (2006) for use with our data. This coding scheme describes six super ordinate categories of metacognitive activity including: orientation, planning, executing, monitoring, evaluation, and elaboration. Within each super ordinate category, Meijer et al. describe an array of more specific activities (e.g., inferring is a specific type of elaboration). Furthermore, Meijer et al. groups these subactivities according to those that are general across tasks, those observed while studying text, and those observed while problem-solving (e.g., inferring is a task general type of elaboration, whereas commenting on problem difficulty is a type of elaboration activity specific to problem-solving tasks).

For the current study, we used all six super ordinate categories described by Meijer, et al. (2006) focusing on specific types of metacognitive activities observed for both general and problem-solving tasks. However, we eliminated specific subcodes in Meijer et al.'s system if they did not fit the task demands of our specific problem-solving situation. For example, one task general planning activity identified by their system was looking for particular information in text. Since we did not have a text in our task this category did not apply and we omitted it from our coding system. Table 1 presents the coding definitions that we used along with examples from our data that reflect these codes.

We coded each transcript for metacognitive activity. The unit of analysis was the speaker turn. If a turn demonstrated more than one type of metacognitive activity it was coded more than once. For each protocol, we calculated the frequency of orientation, planning, executing, monitoring, evaluation, and elaboration activities, as well as the sum of all turns demonstrating one or more types of metacognitive activity. We then converted these frequencies into percentages for ease of comparison.

To calculate percent of overall metacognitive activity, we summed the total number of turns coded and divided by the total number of turns in the transcript. To calculate percent of different metacognitive activity types, we divided the sum of each type by the total number of codes for each transcript. We compared the two conditions for percent of overall metacognitive activity and percent of the six super ordinate metacognition codes of orientation, planning, executing, monitoring, evaluation, and elaboration.

To check reliability, two raters independently coded two randomly selected protocols from the IW condition and two from the TW condition for the six main metacognitive activities (executing, elaboration, evaluation, monitoring, orienting, and planning). We used Cohen's Kappa statistic to assess inter-rater agreement. There was moderately high 
Table 1 Coding definitions and examples

\begin{tabular}{lll}
\hline Code & Definition & Examples \\
\hline Orientation & Activating prior knowledge & M: He is on Timentin. [repeating] \\
& Establishing task demands & N: So he is not on IV for fluids \\
& Hypothesizing & M: How sick does the patient look? \\
& Identifying/repeating important information & \\
& Studying or rereading & \\
\cline { 2 - 2 } & Filling in values/establishing givens & \\
& Looking for particular information & P: Does he have a history of renal \\
& & dysfunction?
\end{tabular}

Subgoaling

Using external source to get explanation

Backward reasoning

Decision to change strategy

Form action plan

Simplify problem

Executing Commenting on explanation

Note-taking, underlining, highlighting

Reacting to question of experimenter

Estimating

Executing action plan

Transferring one representation into another

Monitoring Checking memory capacity

T: I don't know

Claiming (partial) understanding

Comprehension failure

Error detection

Found required information

Information required not found

Noticing inconsistency, confusion, checking plausibility

Noticing unfamiliar words or terms

Noticing retrieval failure

Commenting on task demands or available time

Claiming progress in understanding

Give meaning to symbols or formulae

Noticing differences

Using former interim outcome

Evaluation Checking

Explaining strategy, justifying

M: We'd like to do [stats] blood glucose

A: I would put the foley in regardless of they told me about the urine output. I would put two large bolus IVs in

O: So we'd like to give him some NS, a bolus of NS

L: I'm just thinking aloud because I...I don't know what to do...

A: I agree (giving him Heparin)

M: OK. What information do we have?

E: He keeps crashing so I would continue with the IV because his blood pressure has been getting low and lower 
Table 1 (continued)

\begin{tabular}{lll}
\hline Code & Definition & Examples \\
\hline & $\begin{array}{l}\text { Finding summaries or analogies } \\
\text { Give up or quit }\end{array}$ & $\begin{array}{c}\text { M: Why would his blood pressure be } \\
\text { dropping? }\end{array}$ \\
$\begin{array}{l}\text { Self-critique } \\
\text { Verifying }\end{array}$ & \\
Concluding & $\begin{array}{l}\text { S: If he has blood loss [he'd be } \\
\text { hemorrhaging] }\end{array}$ \\
Connecting & E: He might crash any minute \\
Inferring & M: He could be getting into sepsis \\
Paraphrase/summarize & \\
\hline
\end{tabular}

agreement in terms of which of the six metacognitive activities were present $(\kappa=.653$, $p=.016$; approximately $69 \%$ agreement). The overall percentage agreement was highest for planning (86\%) and least for evaluating (57\%). The mean percent of the metacognitive activities engaged in for the four protocols is presented in Table 2. Given that metacognitive activities were verbal acts shared with team members our assumption was that these actions reflect a degree of co-regulation. We include in our analysis segments from the TW and IW discourse that we examine from Volet's stance of coregulation, where we examine her dimensions of content and social interaction.

\section{Results}

Metacognitive activities and their relationship to patient management

Although both collaborative conditions demonstrated metacognitive activity, the technology condition engaged in a greater percentage (44\%) of it than the TW condition (29\%). In addition, the pattern and timing of metacognitive activity varied. We examined the TW and IW conditions in terms of the distribution of metacognitive activities they engaged in early, in the middle, and towards the end of the session. The IW teams engaged in substantially more planning and orienting than the TW teams in the early stages of problem solving and more execution of strategies later in the problem solving activity. All aspects of metacognition are important but we know that planning and orienting are needed for successful execution of actions leading to effective outcomes (Pintrich 2004; Veenman et al. 2006; Winne and Hadwin 2008; Zimmerman 2006). In this study, technology supported

Table 2 Percent of metacognitive activities in protocols for Traditional Whiteboard (TW) and Interactive Whiteboard (IW) groups

\begin{tabular}{lccccccc}
\hline & Execute & Elaborate & Evaluate & Monitor & Orient & Plan & Total \\
\hline TW & $6.7 \%$ & $1.7 \%$ & $0.5 \%$ & $6.7 \%$ & $1.4 \%$ & $12.1 \%$ & $29.1 \%$ \\
IW & $8.2 \%$ & $2.8 \%$ & $1.8 \%$ & $9.6 \%$ & $6.4 \%$ & $15.5 \%$ & $44.3 \%$ \\
Overall & $7.4 \%$ & $2.3 \%$ & $1.1 \%$ & $8.1 \%$ & $3.8 \%$ & $13.8 \%$ & \\
\hline
\end{tabular}


planning and orienting early on in the problem solving activity, led to higher levels of execution later in the activity (11.5\% vs. $3.6 \%$ in TW) (see Table 3 ). Execution in this study led to effective patient management (following the emergency algorithm of checking airways, breathing, circulation, drugs, endrocine/electrolyte, fever, and general, providing the correct medication, conducting appropriate diagnostic tests, etc.). In an earlier paper pertaining to this work we reported that in the early stages of decision making when the patient situation was less urgent, the IW condition put more effort into interpreting the patient situation (determining patient status based on patient history, test results, patient reactions, etc.) but less into managing the patient than the TW condition did (xxxx). In the late stage, when the patient's situation had become more urgent, the IW condition put more effort into managing the patient than into interpreting the situation.

Examining the type and timing of metacognitive activities in the two conditions demonstrate that technology led to discourse differences that revealed higher metacognitive activity as well as differences as to when such activity occurred. This discourse also revealed differences in co-regulation. We provide examples below of excerpts from teams in both conditions to demonstrate the type of co-regulation occurring in the two conditions along with examples of how the IW annotations were shared and used by others to build a shared understanding of how to manage the patient in distress. Unfortunately, we do not have data on what the TW teams shared verbally prior to acting in the decision-making role in the activity and cannot compare the two conditions on such data. However, we provide some data below to provide readers with a snapshot of the types of annotations that were made using the IW and how they fostered co-regulation.

\section{Pattern of co-regulation in both conditions}

Both conditions were encouraged to discuss the deteriorating patient activity among themselves, however, when problems occurred discussions sometimes came to a halt. This was more obvious in the TW condition. For example, students B and G had different plans for the patient, B told the nurse to check the patient's ' $E C G$ ' and $\mathrm{G}$ proposed giving the patient 'Dextrose'. The teacher (T) intervenes below.

T: You better discuss among yourselves. I mean one of you is telling me dextrose and the other is telling me ECG. You guys have to decide.

(B \& G Confer)

B: So? (To G)

G: Is dextrose ok with you guys? (To B) $5 \%$ with saline.

B: Yes, I think we could give him dextrose.

Table 3 Percent of metacognitive activities in protocols for Traditional Whiteboard (TW) and Interactive Whiteboard (IW) groups early, middle, and late in the session

\begin{tabular}{|c|c|c|c|c|c|c|c|c|c|c|c|c|}
\hline & \multicolumn{2}{|c|}{ Execute } & \multicolumn{2}{|c|}{ Elaborate } & \multicolumn{2}{|l|}{ Evaluate } & \multicolumn{2}{|c|}{ Monitor } & \multicolumn{2}{|l|}{ Orient } & \multicolumn{2}{|l|}{ Plan } \\
\hline & TW & IW & TW & IW & TW & IW & TW & IW & TW & IW & TW & IW \\
\hline Early & $9.3 \%$ & $7.8 \%$ & $2.1 \%$ & $2.6 \%$ & $1.6 \%$ & $0.5 \%$ & $5.2 \%$ & $9.3 \%$ & $1.0 \%$ & $9.8 \%$ & $10.6 \%$ & $22.8 \%$ \\
\hline Middle & $7.1 \%$ & $5.6 \%$ & $0.5 \%$ & $3.1 \%$ & $0 \%$ & $3.6 \%$ & $7.1 \%$ & $12.8 \%$ & $0.5 \%$ & $6.7 \%$ & $21.0 \%$ & $10.3 \%$ \\
\hline Late & $3.6 \%$ & $11.5 \%$ & $2.6 \%$ & $2.9 \%$ & $1.5 \%$ & $1.1 \%$ & $7.7 \%$ & $6.3 \%$ & $3.1 \%$ & $2.3 \%$ & $4.6 \%$ & $13.2 \%$ \\
\hline Total & $6.67 \%$ & $8.30 \%$ & $1.73 \%$ & $2.87 \%$ & $1.03 \%$ & $1.73 \%$ & $6.67 \%$ & $9.47 \%$ & $1.53 \%$ & $6.27 \%$ & $12.07 \%$ & $15.43 \%$ \\
\hline
\end{tabular}


B and $\mathrm{G}$ had a brief communication after the teacher's instructions because they had different proposals for the patient. However, the discussion was very brief. B asked G's opinion who then gave his proposal which B immediately accepted. A similar pattern occurred later on when $\mathrm{G}$ and $\mathrm{B}$ talked about giving more liquid. Using a co-regulation framework we do not see evidence of explanation or synthesis or ideas, rather we see independent individuals coming up with their own ideas and no argument. Instead, the dominant individual's idea is accepted without debate. There is no evidence of elaborating, interpreting, reasoning, building on ideas, explaining in one's own words, or help seeking for understanding. There was little social regulation or co-regulation with verbal contributions from multiple group members. The discussion was brief with little argumentation. Even though $\mathrm{G}$ and $\mathrm{B}$ disagreed initially, they came to an agreement with little negotiation. Similar patterns were identified in the rest of the problem solving episodes. Their discussion was not spontaneous and was often prompted by teacher interventions.

On the other hand the IW discussions involved more turn taking and students contributed more equally in terms of offering ideas. IW students expressed their opinions and the teacher seldom interrupted or pushed them to collaborate. Their discussion was led by ideas from annotations. We provide a chronological look at how the teams demonstrated co-regulation in the sequence of activities below. We provide the first team's discourse, followed by the annotations made by other teams, and finally how the last team incorporated the information from previous teams in their discourse.

The teacher introduces the scenario "Saturday evening, 9 PM, you are asked to see an 80 year old male who was admitted to your floor. The gentleman has hypertension, type II diabetes, Polymyalgia rheumatica PMR. He was admitted for a lower left lobe pneumonia. And the nurse asks you see the patient, because... I am the nurse. ...he looks "sick". So first group, I am the nurse, you are the doc, what you are going to do? You can discuss among yourselves if you want and help each other." After some preamble the first team (M, P) proposes the following to the instructor $(\mathrm{J})$ :

M: Yeah, let's put him on oxygen. His O2 SAT is $95 \%$, but his blood pressure is low and his heart beat is low. High, sorry, his heart rate is high. (laughing) $\mathrm{He}$ is tachycardiac, so we..

P: Yeah.

M: O2 for start?

P: Yeah.

J: So you start treatment right away with oxygen.

M: Yes.

J: How much oxygen? Doctor.

M: He's sounding at $95 \%$ for starters. Let's start with... (Pause \& Laughter)

P: Nasal prongs.

M: No, we give it by mask. The nasal prongs, he is...

J: Would it help if I told you he also had a chronic lymphosemic leukemia?

(General Laughter)

P: Nasal prongs.

M: By mask. If he's sick. The nurse says he's sick. Mind you his SAT is OK, 95,

P: Yeah,

M: (Maybe) nasal prongs.

P: Yeah, sure. 
$\mathrm{J}$ : Nasal prongs,

M: 51.

J: 51 per minute. Anything else that you would do doctor?

As team 1 verbalizes their decision-making the instructor documents their argument shown in Fig. 2. The figure demonstrates what has been documented on the whiteboard with respect to their decisions at this time. Their decisions "O2 5L NP" refers to their giving the patient oxygen, 5 1, with Nasal Prongs. Later in the discourse they ask to "Bolus 500 cc NS" which is documented as well.

When teams were observers they used the interactive whiteboard to record plans for collecting data and for managing the patient. They did not rush to post their ideas right away. If they found that the role-playing team later expressed the same idea, they would erase their original annotation. If they thought their ideas might be useful, they would post them for others to see. Team 2 and 3 annotate the whiteboard with their own information. Team 2 proposes a different opinion on giving oxygen (see Fig. 3). In the excerpt below when team 1 mentioned giving oxygen to the patient, team 2 wrote '2 1 nasal (1)', to suggest giving oxygen with nasal prongs at a speed of 21 . Team 2 did not post it because team 1 continued to argue for giving oxygen right away. Team 1 eventually decided to give oxygen by nasal prongs and team 2 erased their annotation. Later on, team 2 wrote '? 21 nasal prongs' to indicate that they did not quite agree with team 1's suggestion of 'giving 51 by nasal prongs.' Team 2 added a question mark to get team 1's attention. This annotation influenced later decision-making. Furthermore, Team 2 and 3 propose alternatives, such as

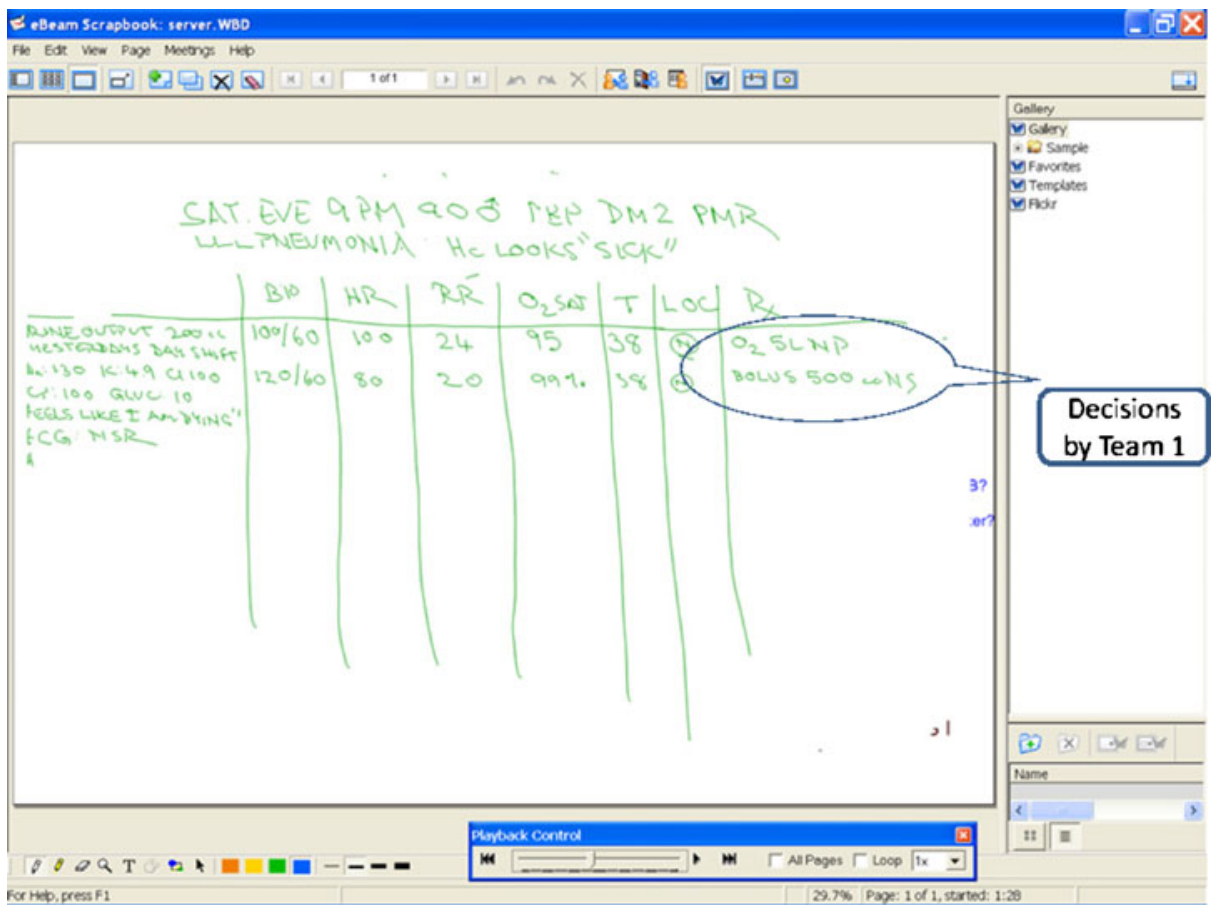

Fig. 2 Screenshot of Team 1 decisions on whiteboard 
"fluid, medication, and blood glucose" (as shown in Fig. 3). These alternative suggestions were taken by Team 1 as shown in the discourse below.

M: Another bolus? OK, Glucose. Another bolus. And do we want to... Ah meds? He could be taking sort of (meds). That could (cause the problem). Has he taken an antibiotic lately that could have... Sounds medications. Complete blood count. OK, is that alright?

P: Hmmm.

M: Sounds like medications could play a thing?

P: Hmmm.

M: Is that alright?

P: Before that we should bolus him again?

M: We should bolus him again. The bolus helped him the first time. So I would bolus him again.

P: And then we can look at what meds he is taking.

$\mathrm{M}$ read the annotations and repeated, i.e., 'glucose', 'another bolus', and 'meds.' $\mathrm{M}$ then began elaborating possible reasons for the problem, such as taking inappropriate medication. P agreed and suggested that they 'access his chart'. $\mathrm{M}$ then proposed to treat the patient with another bolus. P did not object but insisted that they should also check the patient's medications. In terms of co-regulation we see discourse that is of high content that builds on previous annotations and discourse, interpreting the discourse in a way that leads to correct decisions about which medications to give, and how to administer oxygen and drugs and elaborations as to why things should be done in the context of the patient needs.

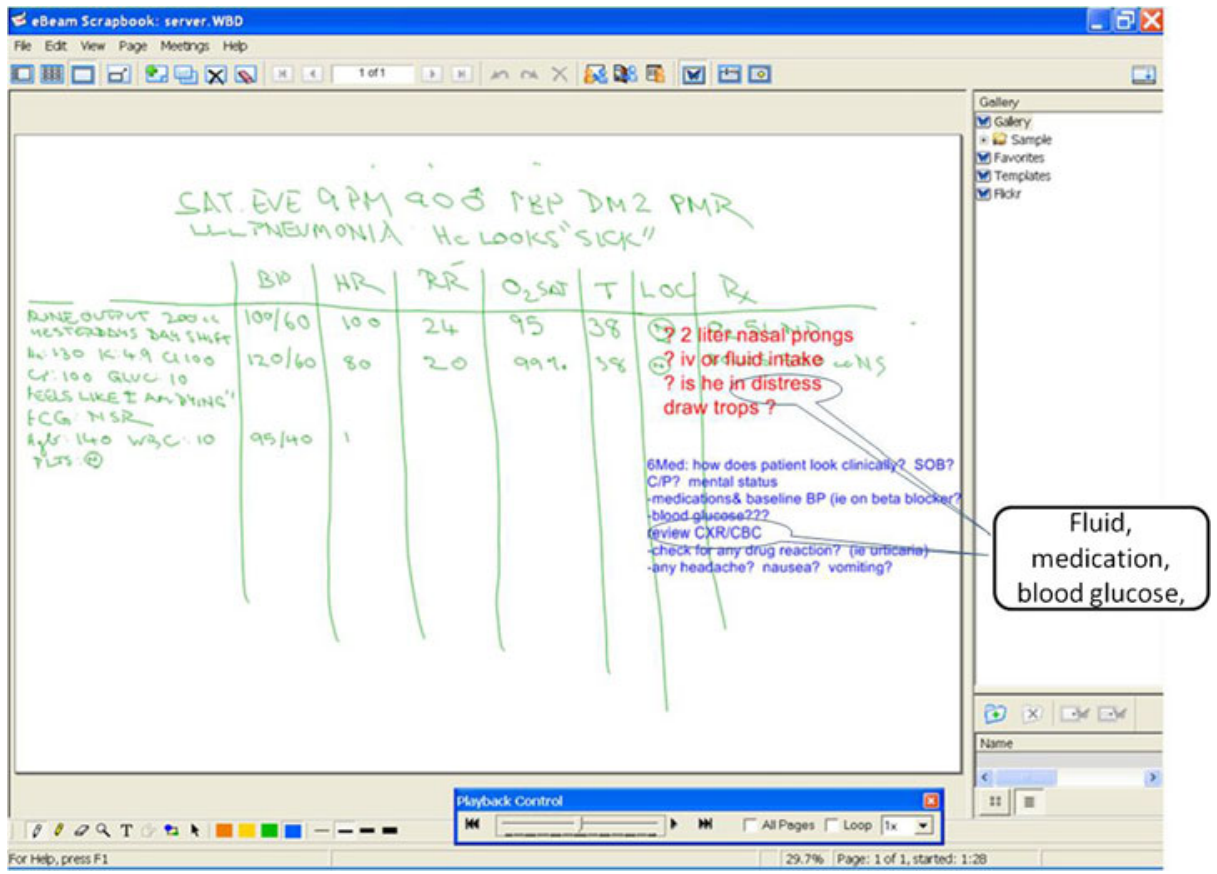

Fig. 3 Screenshot of Team 2 and 3 entries on whiteboard 
There is also evidence of multiple participants, those that are present face to face, and those who have shared information through technology, that are participating in sharing their understanding of the patient problem. Although this is simple case study data, it does provide evidence of collaborative argumentation that is linked to the opinions of the teams engaging in the whiteboard condition. These arguments led to successful decision making in terms of what treatments were need to stop the patient from deteriorating further. The annotations that were shared became part of the learning environment for all teams given they were externalized for the entire class to reflect on. Consequently, it helped all participants in this dynamic role-play where decision making led to better patient management. As the problem becomes more difficult, students consulted and referred to the annotations more, becoming more open to the opinions of others.

\section{Discussion}

The results support our prediction that technology led to more effective forms of coregulation. Although both collaborative conditions engaged in metacognitive activity the technology supported group had higher frequencies of such activity. The pattern and timing of metacognitive subtypes varied. Specifically, the IW condition engaged in more planning and orienting than the TW condition at the outset of the problem that enabled them to engage in more efficient consensus earlier in the activity about what they knew or did not know which assisted them in executing their plans more effectively than the TW $\mathrm{s}$. We interpret this data to suggest that these metacognitive activities led to co-regulatory actions, where high level content knowledge was discussed and multiple partners engaged in dialogue that resulted in common ground early on in the simulation activity. Communications early on in the activity served to establish a shared mental model of the task (Cannon-Bowers et al. 1993; Klein et al. 1993; Orasanu 2005) and basically reduced the need for explicit discussion of issues later in the activity since common ground had been established. Common ground in this context referred to planning and collecting the appropriate patient data. The high presence of orienting dialogue is one indicator of establishing common ground. Orienting refers to such things as activating prior knowledge, establishing task demands, studying or rereading question carefully, hypothesizing, identifying and repeating important, and establishing givens. For example, students may determine what medications the patient is on, and how the medications are administered. These types of activities are especially important in small group activities where common ground must be established prior to taking actions. We found that as the IW teams decreased their orienting in the later stages of the activity they increased their execution skills where they executed action plans to manage the patient. For example,

M: We'd like to do [stats] blood glucose.

A: I would put the foley in regardless of they told me about the urine output. I would put two large bolus IVs in.

O: So we'd like to give him some NS, a bolus of NS,

On the other hand, the TW teams were still engaging in a great deal of orienting in the late stages of the activity, demonstrating that they were still having some difficulty establishing common ground which led to fewer execution actions in the late stage of problem solving. The TW collaboration was less balanced and consequently more time was required communicating orienting issues throughout the activity, which interfered with 
taking appropriate actions when the situation became more urgent ( $\mathrm{xxxx}$ ). We point out that other researchers support the finding that timing of specific self-regulation processes is critical to successful outcomes. Planning processes, such as activating prior knowledge, has a strong relationship to various learning outcomes (e.g., Azevedo and Witherspoon 2009; Greene and Azevedo 2009).

The added value of the technology tools was the facilitation of co-regulation in terms of orienting the team to the task at hand. Orienting is especially important for teams who are working in high stress situations where decisions need to be made quickly and accurately. Trust needs to be established, which requires trust in the cognitive content that is shared as well as trust at the affective level, where the team trusts each other to work in a safe environment, making decisions that are good for both the patient and the team itself. Whereas the TW group had access to an externalization of the problem list that was documented on the whiteboard, the IW tools allowed groups to interact with the development of the problem list dynamically. In the IW condition the teams were able to share information face-to-face as well as through their connected laptops and whiteboard where they were free to make additions and deletions to the medical argument at any time. The TW had face-to-face interactions and access to the traditional whiteboard but did not have the technology tools. The IW served as a cognitive tool to support the building of a medical argument as well as the sharing of the argument building. Although the traditional whiteboards served to visualize the team's argument it did not foster interaction with the documented argument. The IW team could interact with the argument, change it to suit their own needs and use it to serve their early orienting and planning so that they could proceed to their decision making about patient management in the emergency situation. Technology helped facilitate common ground early on in the emergency situation, which is crucial to foster both communication and appropriate actions for patient management. Examining both the types and timing of metacognitive activities can help us identify the points where co-regulation occur and where they might be scaffolded.

Author Notes The authors would like to acknowledge the assistance of Dr. Marguerite Roy in coordinating the metacognitive coding efforts, along with Mr. John Ranelucci and Ms. Ilian Cruz-Panneso for their coding efforts. We also acknowledge Mr. Eric Poitras for his careful editorial advice. We acknowledge Dr. Jeffrey Wiseman for his valuable input as the designer and instructor of the Deteriorating Patient activity and his students for participating in this research. We also acknowledge the granting agency, Social Sciences and Humanities Research Council of Canada for supporting this research.

\section{References}

Azevedo, R., \& Witherspoon, A. M. (2009). Self-regulated use of hypermedia. In D. J. Hacker, J. Dunlosky, \& A. C. Graesser (Eds.), Handbook of metacognition in education (pp. 319-339). New York: Routledge.

Azevedo, R., Cromley, J. G., \& Seibert, D. (2004). Does adaptive scaffolding facilitate students' ability to regulate their learning with hypermedia. Contemporary Educational Psychology, 29, 344-370.

Baker, L., \& Brown, A. L. (1984). Metacognitive skills and reading. In P. D. Pearson (Ed.), Handbook of reading research (pp. 353-394). New York: Longman.

Baker, D. P., Salas, E., King, H., Battles, J., \& Barach, P. (2005). The role of teamwork in the professional education of physicians: current status and assessment recommendations. Joint Commission Journal on Quality and Patient Safety, 31, 185-202.

Bandura, A. (1982). Self-efficacy mechanism in human agency. The American Psychologist, 37, $122-147$.

Bandura, A. (1986). Social foundations of thought and action: A social cognitive theory. Englewood Cliffs: Prentice-Hall. 
Bartsch, K., \& Wellman, H. M. (1995). Children talk about the mind. New York: Oxford University Press.

Boekaerts, M., Pintrich, P., \& Zeidner, M. (Eds.). (2000). Handbook of self-regulation. San Diego: Academic.

Bowers, C. A., Morgan, B. B., Salas, E., \& Prince, C. (1993). Assessment of coordination demand for aircrew coordination and training. Military Psychology, 5, 95-112.

Brown, J. S., Collins, A. \& Duguid, S. (1989). Situated cognition and the culture of learning. Educational Researcher, 18(1), 32-42.

Butler, D., \& Winne, P. H. (1995). Feedback and self-regulated learning: a theoretical synthesis. Review of Educational Research, 65, 245-281.

Cannon-Bowers, J. A., Salas, E., \& Converse, S. (1993). Shared mental models in expert team decision making. In N. J. J. Castellan (Ed.), Individual and group decision making (pp. 221-246). Hillsdale: Erlbaum.

Corno, L., \& Mandinach, E. B. (1983). The role of cognitive engagement in classroom learning and motivation. Educational Psychologist, 18(2), 88-108.

Dinsmore, D. L., Alexander, P. A., \& Loughlin, S. M. (2008). Focusing the conceptual lens on metacognition, self-regulation, and self-regulated learning. Educational Psychology Review, 20, 391409.

Flavell, J. H. (1971). First discussant's comments: what is memory development the development of? Human Development, 14, 272-278.

Greene, J. A., \& Azevedo, R. (2009). A macro-level analysis of SRL processes and their relations to the acquisition of a sophisticated mental model of a complex system. Contemporary Educational Psychology, 34(1), 18-29.

Hacker, D. J., \& Bol, L. (2004). Metacognitive theory: Considering the social-cognitive influences. In D. M. McInerney \& S. v. Etten (Eds.) Big theories revisited (pp. 275-297). Information Age Publishing.

Iiskala, T., Vauras, V., Lehtinen, E., \& Salonen, P. (2011). Socially shared metacognition of dyads of pupils in collaborative mathematical problem-solving processes, Learning and Instruction, 21, 374-393.

Johnson, D. W., \& Johnson, R. T. (Eds.). (1999). Learning together and alone: Cooperative, competitive, and individualistic learning (5th ed.). Boston: Allyn \& Bacon.

Joint Commission on Accreditation of Healthcare Organizations (2006). Sentinel Event Statistics, 30.

Khoshafian, S., \& Buckiewicz, W. (1995). Introduction to groupware, workflow, and workgroup computing. Toronto: Wiley.

Klein, G. A., Orasanu, J., Calderwood, R., \& Zsambok, C. E. (1993). Decision making in action: Models and methods. Norwood: Alex.

Lajoie, S. P. (2008). Metacognition, self regulation, and self-regulated learning: a rose by any other name? Educational Psychology Review, 20, 469-475.

Lories, G., Dardenne, B., \& Yzerbyt, V. Y. (1998). From social cognition to metacognition. In V. Y. Yzerbyt, G. Lories, \& B. Dardenne (Eds.), Metacognition: Cognition and social dimensions (pp. 1-15). London: Sage.

Lu, J., \& Lajoie, S. P. (2008). Supporting medical decision making with argumentation tools. Contemporary Educational Psychology, 33, 425-442.

McCaslin, M. (2004). Coregulation of opportunity, activity, and identity in student motivation. In D. McInerney \& S. Van Etten (Eds.), Big theories revisited, Vol. 4 (pp. 249-274). Greenwich: Information Age.

Meijer, J., Veenman, M. V. J., \& van Hout-Wolters, B. H. A. M. (2006). Metacognitive activities in textstudying and problem-solving: development of a taxonomy. Educational Research and Evaluation, 12 (3), 209-237.

Olekalns, M., \& Smith, P. L. (2005). Moments in time: metacognition, trust, and outcomes in dyadic negotiations. Personality and Social Psychology Bulletin, 31(12), 1696-1707.

Orasanu, J. (2005). Crew collaboration in space: A naturalistic decision-making perspective. Aviation, Space, and Environmental Medicine, 76(6), II, Supplement B154-163.

Paris, S. G., \& Paris, A. H. (2001). Classroom applications of research on self-regulated learning. Educational Psychologist, 36(2), 89-101.

Pintrich, P. R. (2004). A conceptual framework for assessing motivation and self-regulated learning in college students. Educational Psychology Review, 16, 385-407.

Reusser, K. (2001). In N.J. Smelser, P. Baltes and F.E. Weine (Eds.), International encyclopedia of the social and behavioral sciences (pp. 2058-2062). Oxford, UK: Pergamon/Elsevier Science.

Roschelle, J., \& Teasley, S. D. (1995). The construction of shared knowledge in collaborative problem solving. In C. E. O’Malley (Ed.), Computer-supported collaborative learning (pp. 69-97). New York: Springer.

Salonen, P., Vauras, M., \& Efklides, A. (2005). Social interaction: what can it tell us about metacognition and coregulation in learning? European Psychologist, 10(3), 199-208. 
Schunk, D., \& Zimmerman, B. (1994). Self-regulation of learning and performance. Hillsdale: Erlbaum.

Veenman, M., Van Hout-Wolters, B., \& Afflerbach, P. (2006). Metacognition and learning: conceptual and methodological considerations. Metacognition and Learning, 1, 3-14.

Volet, S., Summers, M., \& Thurman, J. (2009). High-level co-regulation in collaborative learning: how does it emerge and how is it sustained? Learning and Instruction, 19, 128-143.

Winne, P. H. (2001). Self-regulated learning viewed from models of information processing. In B. J. Zimmerman \& D. Schunk (Eds.), Self-regulated learning and academic achievement: Theoretical perspectives (pp. 153-189). Mahwah: Erlbaum.

Winne, P., \& Hadwin, A. (2008). The weave of motivation and self-regulated learning. In D. Schunk \& B. Zimmerman (Eds.), Motivation and self-regulated learning: Theory, research, and applications (pp. 297-314). Mahwah: Erlbaum.

Winne, P. H., \& Perry, N. E. (2000). Measuring self-regulated learning. In M. Boekaerts, P. Pintrich, \& M. Zeidner (Eds.), Handbook of self-regulation (pp. 531-566). San Diego: Academic.

Wiseman, J., \& Snell, L. (2008). The deteriorating patient: a realistic but 'low-tech' simulation of emergency decision-making. The Clinical Teacher, 5, 93-97.

Wright, M. C., Phillips-Bute, B. G., Petrusa, E. R., Griffin, K. L., Hobbs, G. W. \& Taekman, J. M. (2009). Assessing teamwork in medical education and practice: Relating behavioural teamwork ratings and clinical performance, Medical Teacher, 31(1), 30-38.

Zimmerman, B. J. (1989). A social cognitive view of self-regulated academic learning. Journal of Educational Psychology, 81, 329-339.

Zimmerman, B. (2001). Theories of self-regulated learning and academic achievement: an overview and analysis. In B. Zimmerman \& D. Schunk (Eds.), Self-regulated learning and academic achievement: Theoretical perspectives (pp. 1-37). Mahwah: Erlbaum.

Zimmerman, B. J. (2004). Sociocultural influence and students' development of academic self-regulation: A social-cognitive perspective. In D. M. McInerney, \& S. v. Etten (Eds.), Big theories revisited. Information Age Publishing.

Zimmerman, B. (2006). Development and adaptation of expertise: The role of self-regulatory processes and beliefs. In K. Ericsson, N. Charness, P. Feltovich, \& R. Hoffman (Eds.), The Cambridge handbook of expertise and expert performance (pp. 705-722). New York: Cambridge University Press.

Zimmerman, B., \& Schunk, D. (2001). Self-regulated learning and academic achievement (2nd ed.). Mahwah: Erlbaum. 